\title{
El amianto en la edificación: variedades y riesgos asociados a las labores de deconstrucción
}

\section{Varieties of asbestos in buildings and risks associated with the work of deconstruction}

$\underline{\text { M. Mateo }}^{(*)}$, C. Pérez-Carramiñana(*), S. Chinchón ${ }^{(*)}$

\section{RESUMEN}

El amianto es un producto mineral que fue ampliamente utilizado como material de construcción durante la segunda mitad del siglo XX. El descubrimiento de las enfermedades producidas por la inhalación de sus fibras impulsaron la creación de normativas para proteger a los trabajadores y prohibir su comercialización.

Este artículo describe los tipos mineralógicos más importantes desde el punto de vista de la construcción, los principales efectos sobre la salud derivados de su exposición, e identifica su presencia en materiales de construcción y su riesgo potencial. Se revisa el proceso de diagnóstico de amianto en edificios existentes, la toma de muestras y la determinación de la concentración de fibras de amianto. Estos datos permiten conocer el grado de friabilidad del material y determinar el tipo de actuación a realizar. Asimismo, se revisa la situación actual de la gestión de residuos de amianto.

\section{SUMMARY}

Asbestos is a mineral product that was widely used as a construction material in the second half of the twentieth century. The discovery of diseases caused by the inhalation of its fibres led to the establishment of regulations to protect workers and prohibit its marketing.

The article describes the most important mineralogical types from the construction point of view, the main effects on health resulting from its exposure, and identifies their presence in building materials and their potential risk. It reviews the process of surveying asbestos in existing buildings, sampling and determination of the concentration of asbestos fibres. This data provides information about the degree of friability of the material and determines what action to take. It also reviews the current status of waste management of asbestos.
Palabras clave: Amianto; friabilidad; diagnóstico; deconstrucción.
Keywords: Asbestos; friability; survey; deconstruction.

\footnotetext{
(*) Escuela de Arquitectura - Universidad de Alicante, (España). 


\section{INTRODUCCIÓN}

Amianto o asbesto son denominaciones de origen griego cuyo significado es amiantoincorruptible y asbesto-inextinguible. Es un producto mineral de gran durabilidad y reducido coste, formado por un conjunto de haces de fibras minerales sólidamente unidas. El amianto confiere excelentes propiedades físicas y químicas a los materiales que lo contienen: resistencia mecánica, resistencia a la abrasión, aislamiento térmico y acústico, baja conductividad eléctrica, incombustibilidad, no biodegradabilidad y resistencia a agentes químicos. Estas propiedades han hecho del amianto un elemento muy utilizado en materiales diversos (estudios arqueológicos demuestran la presencia de fibras de amianto en piezas cerámicas finlandesas de 2500 años A.C.) y muy concretamente en la industria de la construcción.

El uso industrial del amianto, todavía a escala moderada, comenzó gracias al descubrimiento de unos depósitos en los montes Urales en el occidente de Rusia, alrededor de 1720. El descubrimiento llevó a la fundación de la primera fábrica de productos de amianto, incluyendo textiles, calcetines, guantes y carteras de mano. En 1827 se inició la manufactorización de ropas para bomberos en Milán.

En 1847 se descubrió en Canadá el primer gran yacimiento de amianto de tipo crisotilo, pero no fue hasta finales de 1860 y la década de 1870 cuando empezó la explotación industrial de las minas (1). El periodo de industrialización definitiva del amianto empezó en Inglaterra en el año 1871 con la creación de la compañía Patent Asbestos Manufacturing Company, Ltd., utilizándose durante todo el siglo XX. En España, el período de máxima utilización fue el comprendido entre los años 1960 y 1984 . A partir de la década 1970-1980, el consumo de amianto en el mundo ha ido disminuyendo al suplirse por otras fibras artificiales.

A medida que el amianto se utilizaba, fueron conociéndose los riesgos que representaba para la salud la inhalación de sus fibras, provocando la prohibición progresiva de sus distintos usos. En 1978 una resolución del Parlamento Europeo declaró el amianto sustancia cancerígena, pero no fue hasta 1983 cuando se publicó la primera directiva sobre el amianto en la Unión Europea (2). En 1999, se prohibió su comercialización y uso, estableciendo una moratoria hasta el año 2005 para que cada país comunitario la incorporara en su legislación (3). Sin embargo, mientras en los países desarrollados se ha hecho efectiva la prohibición del amianto, en los países en vías de desarrollo los procesos de extracción, procesado y uso del amianto van en aumento (4).

Todos los tipos de amianto están clasificados como cancerígenos de categoría A1. Aún así, su presencia en los materiales de construcción no supone directamente una situación de riesgo. Las fibras del amianto no se desprenden de manera natural sino que es necesario que se produzca una manipulación o alteración de dichos materiales para que tenga lugar la liberación y emisión de las fibras. Los materiales con amianto (MCAs o ACMs, asbestos-containg materials, en inglés), si son materiales no friables, pueden seguir instalados y en uso sin que sea necesario realizar ninguna intervención, aunque se deberá redactar un Plan de control periódico que establezca inspecciones para evaluar su estado de conservación (5).

\section{VARIEDADES Y EFECTOS SOBRE LA SALUD}

\subsection{Tipos mineralógicos más importantes desde el punto de vista de la industria de la construcción}

El asbesto o amianto es un mineral metamórfico, constituido por silicatos de variada composición con estructura fibrosa y aspecto sedoso, más o menos flexible. Las fibras de amianto más empleadas en aplicaciones de construcción pertenecen a dos grupos mineralógicos: las serpentinas y los anfíboles.

En lo que sigue a continuación se hace una breve descripción de los minerales de asbesto empleados en construcción y se reserva un apartado final para ilustrar su morfología y describir el modo de analizarlos.

\subsubsection{Grupo serpentina:}

- Crisotilo: Conocido también como amianto blanco, aunque en ocasiones presenta un tono ligeramente verdoso. Es un silicato de magnesio hidratado que responde a la fórmula $3 \mathrm{MgO} .2 \mathrm{SiO} 2.2 \mathrm{H} 2 \mathrm{O}$. Se caracteriza por sus fibras curvadas, flexibles y finas, sedosas, fáciles de hilar y resistentes al calor pero no a los ácidos. Constituye más del 90\% del amianto utilizado en la construcción. Es el que menor toxicidad presenta (6).

\subsubsection{Grupo anfíboles:}

- Crocidolita: Conocida también como amianto azul debido a su color. Es un silicato hidratado de hierro y sodio de composición compleja y variable al que podemos 
asignar la fórmula $3 \mathrm{H} 2 \mathrm{O} .2 \mathrm{Na} 2 \mathrm{O} .6(\mathrm{Fe}, \mathrm{Mg})$ $\mathrm{O} .2 \mathrm{Fe} 2 \mathrm{O} 3.17 \mathrm{SiO} 2$. Se presenta en forma de fibras rectas, largas y finas de color azul o azul verdoso. Es muy resistente a los ácidos y se ha utilizado para la fabricación de tubos a presión en fibrocemento, como aislante ignífugo en construcción y como refuerzo de plásticos y carcasas de baterías. Es el más tóxico de todos los minerales de amianto (7).

- Amosita: Conocida también como amianto marrón. Es un silicato hidratado de magnesio y hierro, también de composición variable, al que se puede asignar la fórmula $\mathrm{FeSiO} 3 . x \mathrm{H} 2 \mathrm{O} / \mathrm{FeO} . \mathrm{SiO} 2$. $\mathrm{xH} 2 \mathrm{O}$. Se caracteriza por sus fibras rectas y largas de color grisáceo o pardusco. Es resistente a los ácidos y al calor y se utiliza para aislamientos térmicos.

Además de los dos compuestos descritos, hay tres variedades de amianto del grupo de los anfíboles con alguna aplicación industrial (antofilita, tremolita y actinolita) que no tienen interés en construcción.

\subsection{Mecanismos de acción. Enfermedades producidas por el amianto}

El riesgo de que se presente una enfermedad asociada al amianto está relacionado con la exposición a sus fibras, viniendo condicionada su peligrosidad por su composición principalmente, aunque es también importante su morfología. El grado de riesgo depende de la concentración de fibras presentes en el aire, la duración y la frecuencia de la exposición, el tamaño de las fibras inhaladas y el tiempo transcurrido desde la exposición inicial.

Las fibras de amianto penetran en el organismo por vía inhalatoria, alcanzando las de menor tamaño $(<3 \mu)$ las vías respiratorias, siendo el resto expulsadas por la saliva y el esputo. Sin embargo, una proporción variable, que depende de la cantidad y tipo de fibras así como de la eficacia de depuración, alcanza el intersticio pulmonar. Las fibras más cortas $(<10 \mu \mathrm{m})$ son fagocitadas o rodeadas por macrófagos, formando granulomas, mientras que las fibras largas mayores de $10 \mu \mathrm{m}$ son recubiertas de ferroproteína, constituyendo el Ilamado cuerpo ferruginoso o cuerpo asbestósico (8). Los cuerpos asbestósicos son los indicadores de la inhalación de fibras de amianto, claves para conocer el grado de exposición.

Después de un período de latencia que suele ser superior a 20 años y que puede ser hasta de 40 ó 50 años, pueden aparecer enfermedades asociadas a la exposición al amianto. Este periodo de latencia en la aparición de la enfermedad hace que, a pesar de la prohibición actual de la comercialización y uso del amianto y, como consecuencia de su extensiva utilización en los años previos, las enfermedades relacionadas con la inhalación de amianto tengan plena vigencia.

Las enfermedades graves más frecuentes relacionadas con el amianto son: la asbestosis (fibrosis pulmonar), el cáncer de pulmón y el mesotelioma (pleural o peritoneal), habiéndose encontrado también asociación con otras neoplasias (carcinomas gastrointestinales o de laringe). Es importante realizar una vigilancia de la salud de los trabajadores expuestos al amianto, ya que permite reconocer el triple de casos de patología por amianto en relación a los declarados espontáneamente (9).

\section{MATERIALES DE CONSTRUCCIÓN CON AMIANTO}

Los materiales con amianto (MCAs) son aquellos en los que el amianto ha sido añadido deliberadamente en su composición. El crisotilo ha venido siendo la forma más utilizada en la construcción y representa el 95\% de la producción mundial. Le siguen en importancia la crocidolita y la amosita, teniendo un uso muy limitado la antofilita, la tremolita y la actinolita.

Debido a que el uso de MCAs está prohibido en los países occidentales, en ellos los encontraremos en edificios construidos con anterioridad a la prohibición del amianto o reparados en su momento con materiales que lo contienen.

\subsection{Clasificación de los materiales con amianto en edificios}

\subsubsection{MCA como producto de construcción}

MCAs como productos aislantes

- Fibras sueltas: Utilizadas para revestir conducciones, como aislante interior de puertas cortafuegos y para rellenar las cámaras de aire en paredes y falsos techos. Prohibidas desde 1994.

- Calorifugado y cordones: Empleados como protección para calderas y conducciones de fluidos a altas temperaturas. Son fibras de amianto trenzado que forran calderas y tuberías para aislarlas. El riesgo de desprendimiento de las fibras no es alto si no se manipulan.

- Mortero proyectado: Utilizado como aislamiento térmico y acústico y para la 
1. Cubierta de fibrocemento.

2. Canalizaciones de agua, bajantes de aguas residuales, canalones, conductos de humos. protección contra incendios de estructuras metálicas de edificios. Consistía en proyectar separadamente y con pistola fibras de amianto y agua a presión mezcladas con cemento. Es una de las aplicaciones más peligrosas porque tiene una friabilidad elevada. Prohibido su empleo desde 1984. En algunos casos ("flocage" de aparcamientos) se ha llegado a encontrar la utilización de la variante crocidolita, con la peligrosidad añadida que comporta. También era utilizado como revestimiento termo-acústico.

\section{MCAs como productos prefabricados}

- Cartón y placas de baja densidad $(<1 \mathrm{~g} /$ $\mathrm{cm}^{3}$ ): Placas ignífugas empleadas para proteger estructuras metálicas, focos puntuales de calor (radiadores o locales con calderas) o la confección de conductos de impulsión de aire, desenfumaje, etc. Empleadas también como placas absorbentes acústicas para falsos techos y divisiones. Mezcladas las fibras con celulosa se fabricaban papeles y cartones aislantes utilizados para el relleno en juntas y huecos. Prohibidos desde 1994.

MCAs como productos de alta resistencia mecánica

- Losetas termoplásticas: Utilizadas como pavimento en interiores. Son pavimentos vinílicos de PVC con un contenido de amianto del $10-25 \%$. El desprendimiento de fibras es improbable durante un uso normal. La posibilidad de desprendimiento se produce al cortar o perforar losetas. Prohibido desde 1994.

- Telas asfálticas: Consistentes en amianto mezclado con betún. Utilizadas para la fabricación de tejados semirrígidos y para la impermeabilización de cubiertas y canalones.

- Plásticos reforzados: Pinturas y másticos con un porcentaje de adición de amianto. Este tipo de pinturas están prohibidas desde 1989 y los másticos, desde 1994.

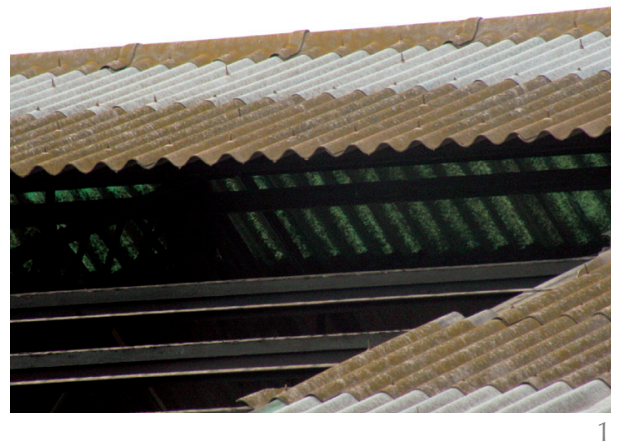

MCAs como productos de fibrocemento

- Fibrocemento: En forma de chapa ondulada para cubiertas (Figura 1), cisternas y depósitos de agua, tubos de alta presión para redes de agua potable, bajantes de aguas residuales y canalones (Figura 2), conductos de humo y shunts. En 1984 se prohibió la utilización de la crocidolita en su fabricación, y en diciembre de 2001 se prohibió la fabricación y comercialización del producto con crisotilo, que empezó a ser efectiva en diciembre de 2002.

\subsubsection{MCA en función del sistema constructivo}

Como componente dentro de un sistema constructivo se puede clasificar como:

- Aplicaciones según la estructura

En estructuras metálicas el amianto se puede encontrar en proyectados, morteros o placas aislantes, ya que durante el período (1974-1984) la mayoría de estructuras metálicas se revistieron con amianto proyectado o con mortero. Aunque las estructuras de hormigón no necesitaban ningún revestimiento por su estabilidad al fuego, excepcionalmente se puede encontrar amianto en aplicaciones de mortero o proyectados de la misma época.

- Aplicaciones según el tipo de instalaciones

Agua climatizada: calorifugaciones en instalaciones de calefacción (1981-1994).

Aires acondicionados: (1950-1994)

Otros: en protecciones eléctricas, cordones, juntas de tuberías, etc (1950-1994).

\subsubsection{MCA en función del uso del edificio}

En función del uso del edificio podemos encontrar los siguientes MCAs:

- Pública concurrencia: paneles acústicos y aislantes (1940-1994), losetas vinílicas (1950-1994), fibras sueltas en cámaras de aire (1920-1984).

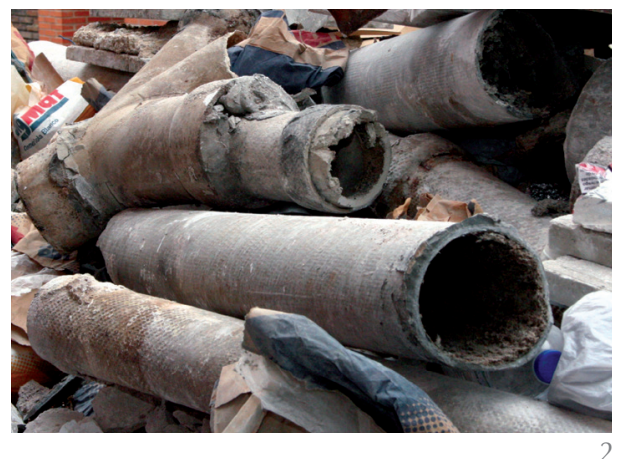


- Aparcamiento: proyectados, morteros, placas y fibras sueltas en cámaras de aire (1968-1984).

- Vivienda: aislamiento térmico en cámaras de aire, cubiertas y fachadas, falsos techos de morteros, paneles aislantes, proyectados o fibras sueltas (1979-1984), también se puede encontrar fibrocemento (principios 1900-2002).

\subsection{Riesgo por la presencia de materiales con amianto}

A pesar de que actualmente esté prohibido el uso, la producción, la comercialización y la instalación de amianto o de productos que lo contengan, el problema reside en el amianto ya instalado y el que se encuentra en forma de residuo. La presencia de MCAs en un edificio no siempre implica riesgo de inhalación de fibras, ya que éste depende de la friabilidad del material, de la protección física del trabajador y del estado de conservación y trabajos realizados sobre el MCA. Actualmente, el riesgo más elevado de exposición al amianto es el de los trabajadores que participan en tareas de deconstrucción y el de los que, durante trabajos de manteniemiento y reparación de edificios, encuentran amianto de improviso.

Los parámetros que determinan la peligrosidad para la salud de la presencia de fibras de amianto son:

- La concentración ambiental, polvo de amianto en el aire (fibras $/ \mathrm{cm}^{3}$ )

- Tipo de fibra de amianto, siendo más peligrosas para la salud las de los anfíboles (crocidolita y amosita) que las serpentínicas (crisotilo), debido a la forma de la fibra en sí.

- La medida de las fibras $(L>5 \mu \mathrm{m} ; \mathrm{d}<3 \mu \mathrm{m}$; $\mathrm{L} / \mathrm{d}>3)$

- Tiempo de exposición a las fibras

- Ritmo respiratorio asociado al esfuerzo físico y a las condiciones termohigrométricas

- Condiciones anatómicas y funcionales de la persona

\subsubsection{Friabilidad del MCA}

La friabilidad es la capacidad que tiene un material de liberar las fibras que contiene, depende del tipo de material y de su composición (10), aumentando cuando el material envejece y se rompe o deteriora. El MCA friable es cualquier material que contiene más de un $1 \%$ de amianto y que, cuando está seco, puede ser desmenuzado, pulverizado o reducido a polvo por presión manual. El MCA no friable es cualquier material que contiene más de un $1 \%$ de amianto y que, cuando está seco, no puede ser desmenuzado, pulverizado o reducido a polvo por presión manual (11).

Los materiales friables son susceptibles de liberar fibras como consecuencia de choques, vibraciones o movimiento del aire. Los materiales no friables no desprenden fibras a no ser que sea por la acción directa de máquinas o herramientas. Los materiales no friables degradados o demolidos pasan a ser materiales friables.

Dentro de los materiales no friables, la Environmental Protection Agency (EPA) de los Estados Unidos de América hace dos distinciones (11):

- Materiales no-friables categoría I. Comprende los aglomerantes, juntas, revestimientos flexibles de suelos y techados asfálticos.

- Materiales no-friables categoría II. Comprende el resto de materiales no friables no incluidos en la categoría II de los cuales el más importante es el fibrocemento.

\subsubsection{Definición tipológica de los edificios de riesgo}

Se clasifican en dos grupos, según si el riesgo puede considerarse medio o alto. Los edificios con aplicaciones de amianto de riesgo medio se caracterizan por el uso de derivados del fibrocemento, empleados hasta finales de los años 90. Los edificios con aplicaciones de amianto de riesgo alto se definen tipológicamente por una serie de parámetros que permiten identificarlos:

- Año de construcción: 1965-1985

- Tipo de estructura: metálica

- Uso edificio: público

- Instalaciones: calefacción central, producción centralizada de agua caliente sanitaria (1).

\section{DIAGNÓSTICO DE AMIANTO EN EDIFICIOS EXISTENTES}

El objeto del proceso de diagnosis es evaluar la presencia de amianto en los edificios existentes. Dada la gran utilización de amianto en materiales de construcción durante las últimas décadas y la problemática que genera su deterioro, diversos países occidentales han dictado normas para el diagnóstico de amianto en edificios: EEUU (12), Reino Unido (13), Francia (14), esta última reflejada en una Nota Técnica de Prevención (15). No existe normativa española, sólo dos Notas Técnicas de Prevención: NTP 632 (10) y NTP 633 (5) que dan unas pautas de actuación en la diagnosis. 
3. Toma de muestras

\subsubsection{Sondeo}

El sondeo es una acción complementaria a la inspección visual (como por ejemplo desmontaje o perforación) que permite llegar a emitir un diagnóstico sin la necesidad de realizar toma de muestras y su posterior análisis. En función del destino del inmueble los sondeos serán no destructivos o destructivos.

\subsubsection{Toma de muestras}

En el caso de ser necesaria la toma de muestras, el técnico decidirá los materiales de los cuales debe extraerse muestra y el número de éstas (Tablas 1, 2 y 3 ) en función de la naturaleza del material, la homogeneidad del mismo y la superficie que tenga el área de muestreo.

Las muestras se deben extraer con herramientas de corte o golpeado manual que desprendan las mínimas fibras al ambiente, evitando en lo posible cualquier herramienta de fricción o abrasión (Figura 3).

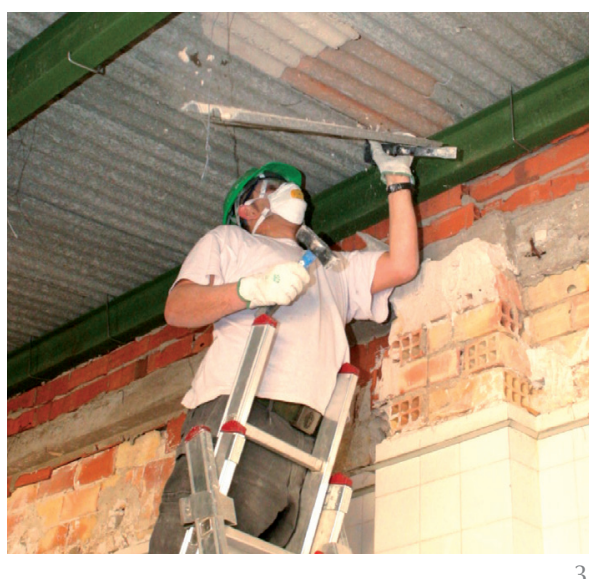

Tabla 1. Número de muestras a extraer según la superficie según la Normativa española

\begin{tabular}{|c|c|c|}
\hline Superficie & Muestras recomendadas & Mínimo número de muestras a extraer \\
\hline Inferior a $100 \mathrm{~m}^{2}$ & 2 & 1 \\
\hline Entre 100 y $500 \mathrm{~m}^{2}$ & 3 & 2 \\
\hline Mayor a $500 \mathrm{~m}^{2}$ & $\begin{array}{c}\text { 2 por cada } 500 \mathrm{~m}^{2} \text { o fracción, } \\
\text { hasta un máximo de 9 }\end{array}$ & $\begin{array}{c}1 \text { por cada } 500 \mathrm{~m}^{2} \text { o fracción, } \\
\text { hasta un máximo de } 9\end{array}$ \\
\hline
\end{tabular}

Fuente: Casanovas, X., Trujillo, L., Freixa, A.. NTP 633: Detección de amianto en edificios (II): Identificación y metodología de análisis. Ministerio de Trabajo y Asuntos Sociales. Instituto Nacional de Seguridad e Higiene en el Trabajo, 2004

Tabla 2. Número de muestras a extraer según la superficie según la Normativa americana

\begin{tabular}{|c|c|c|}
\hline Superficie & Muestras recomendadas & Mínimo número de muestras a extraer \\
\hline Inferior a $92,90 \mathrm{~m}^{2}$ & 9 & 3 \\
\hline Entre 92,90 y $464,51 \mathrm{~m}^{2}$ & 9 & 5 \\
\hline Mayor a $464,51 \mathrm{~m}^{2}$ & 9 & 7 \\
\hline
\end{tabular}

Fuente: EPA 560-5-85-018. Asbestos in Buildings: Simplified Sampling Scheme for Friable Surfacing Materials. EPA (U.S. Environmental Protection Agency), 1985.

Tabla 3. Número de muestras a extraer según la superficie y el tipo de material según la Normativa británica

\begin{tabular}{|c|c|c|}
\hline Tipo de material & Superficie & Muestras recomendadas \\
\hline Proyectados & Mayor a $100 \mathrm{~m}^{2}$ & $1 \mathrm{cada} 25-30 \mathrm{~m}^{2}$ \\
\hline \multirow{2}{*}{ Calorifugados } & Menor de $20 \mathrm{~m}$ & $1 \mathrm{cada} 3 \mathrm{~m}$ \\
\cline { 2 - 3 } & Mayor de $20 \mathrm{~m}$ & $1 \mathrm{cada} 6 \mathrm{~m}$ \\
\hline Aislamiento & - & 1 cada $25 \mathrm{~m}^{2}$ \\
\hline Cemento - amianto & - & 1 por cada tipo de material \\
\hline
\end{tabular}

Fuente: HSG264, Asbestos: The survey guide. Health and Safety Executive Books, Sudbury, 2010 
Cada muestra se debe colocar en una bolsa o recipiente hermético con una etiqueta que indique el número de la muestra y el lugar de donde se ha extraído, para luego remitirlas a un laboratorio. Si bien, cabe destacar que en España no hay laboratorios acreditados para el análisis de amianto en materiales. Los laboratorios acreditados por el Ministerio de Trabajo son para el análisis de fibras de amianto en aire.

Tras la toma de muestras se evaluará la cantidad de polvo generado para decidir las precauciones a tomar. La zona de donde se ha extraído la muestra se deberá fijar con productos encapsulantes que eviten la dispersión de las fibras. Asimismo, tanto el material empleado para la extracción como la zona de actuación han que quedar limpios, y todos los residuos, ropa y mascariIlas desechables deben tratarse como residuos contaminados con amianto.

\subsubsection{Informe de diagnóstico de amianto en edificios}

El proceso de diagnosis encaminado a evaluar la presencia de amianto en los edificios existentes debe permitir escoger el sistema de intervención más apropiado en cada caso, eligiendo entre la no intervención con inspecciones periódicas, la estabilización, el confinamiento o la eliminación de los materiales que contienen amianto (MCAs) (16).

Con los resultados obtenidos se realiza un informe en el que se incluyen las condiciones de la inspección, resultados y conclusiones así como anexos con planos, análisis de laboratorio, estado de conservación de los materiales y condiciones de seguridad. El informe debe establecer las recomendaciones más adecuadas en función del resultado de la diagnosis, concluyendo con la necesidad o no de realizar algún tipo de intervención.

La "No Intervención" se aconseja cuando los resultados muestran que la probabilidad que pasen las fibras al aire durante el uso normal del edificio es muy baja, el material está en buen estado y el contenido de fibras en el aire se encuentra dentro de los límites permitidos. Cuando el material está en buen estado pero se puede degradar accidentalmente, puede no realizarse ninguna intervención pero se han de programar unas visitas periódicas.

En el caso de "Intervención", antes de empezar se establecerá un plan de trabajo. Existen dos tipos de intervención: tratamiento y retirada. El tratamiento se efectúa cuando el material que contiene amianto no está degradado y se desea asegurar su durabilidad, manteniendo el material en su lugar y evitando la dispersión de fibras en el ambiente. Dentro de las operaciones de tratamiento, encontramos las labores de "Estabilización", donde se aplican sobre el MCA una serie de capas de resina, endurecedor superficial y film elastómero y las de "Confinamiento", donde se reviste el MCA. La retirada se escoge cuando el MCA está muy degradado o cuando está situado en un espacio incompatible con su uso.

\subsection{Metodología de evaluación de fibras de amianto}

La determinación de fibras de amianto permite identificar los MCAs, evaluar y controlar la exposición a fibras de los trabajadores que los manipulan y evaluar la contaminación ambiental por fibras de amianto. Las muestras y el procedimiento de análisis para cada uno de los casos son diferentes y específicos. En el primer caso, las fibras de amianto se analizan en muestras de materiales. En los otros dos casos, se requiere determinar la concentración de las fibras de amianto en el aire.

\subsubsection{Identificación de fibras de amianto en el material}

Para confirmar la presencia de amianto en los materiales, las muestras extraídas se deben enviar a un laboratorio que disponga de las técnicas analíticas adecuadas. La identificación de las fibras de amianto debe basarse en la siguiente secuencia analítica:

1. Se realiza un examen preliminar visual de la muestra para determinar el tipo del que se trata y el tratamiento (si procede) que sea necesario.

2. Se trata la muestra (si es necesario) para desprender o aislar las fibras. Se realiza un examen detallado y completo en el microscopio estereoscópico para clasificar los tipos presentes de fibras.

3. Las fibras representativas se preparan para analizar con la metodología que se haya escogido. (En el Apartado 6 se describen los métodos más habituales de análisis).

Tras la obtención de los resultados del análisis de las muestras en laboratorio, se procede a su clasificación, separando las que contienen amianto de las que no. Si contienen amianto se determina su grado de friabilidad. Si son no friables no será necesario realizar ninguna intervención pero sí se deberá redactar un plan de control periódico. Si los materiales analizados son friables, se evaluará el riesgo potencial de exposición a fibras y se actuará en consecuencia con el tipo de tratamiento más adecuado. 


\subsubsection{Evaluación del riesgo potencial}

A partir de los resultados del laboratorio, aquellas muestras que contengan amianto y sean friables deberán ser analizadas para poder realizar el diagnóstico final. Dicho análisis consistirá en evaluar la posibilidad de que las fibras de amianto sean liberadas al aire y la posibilidad de exposición a estas fibras. Estas premisas quedan englobadas en el concepto de Evaluación del Riesgo Potencial.

La NTP 633 establece una serie de parámetros para evaluar el riesgo potencial. Una vez valorado cada uno de los factores se llega a un resultado numérico que determinará si es necesario intervenir en el MCA o no.

\subsubsection{Determinación de la concentración de fibras de amianto en el aire. Evaluación ambiental}

La evaluación ambiental tiene como objetivo la medida de la posible contaminación del aire por fibras de amianto para estimar si existe riesgo para las personas. Las mediciones ambientales han de realizarse en el caso de encontrar MCAs friables con riesgo potencial de que sus fibras de amianto sean liberadas al aire. Para saber si existe riesgo de respirar fibras, debe efectuarse una valoración ambiental mediante una serie de muestreos comprobando que los resultados se encuentren dentro de los valores límite ambientales de exposición al amianto $\left(\mathrm{f} / \mathrm{cm}^{3}\right)$.

La determinación de la concentración de fibras de amianto debe realizarse mediante métodos que permitan la comparación de resultados. Existen dos técnicas analíticas aplicables a la determinación de fibras de amianto en aire: Microscopía Óptica y Microscopía electrónica (barrido y/o transmisión). La utilización de una u otra o de varias a la vez estará en función, aparte de la disponibilidad instrumental en el laboratorio, del tipo de análisis o información deseada (identificación del tipo de fibras y/o cuantificación de la concentración de fibras), así como de la complejidad de la muestra.

La normativa europea recoge que para la medición del amianto en el aire se tendrán en cuenta únicamente las fibras con una longitud superior a $5 \mu \mathrm{m}$, una anchura inferior a $3 \mu \mathrm{m}$ y cuya relación longitud/anchura sea superior a 3:1 (2). Asimismo, se indica que el recuento de las fibras se efectuará preferentemente mediante PCM (microscopio con dispositivo para contraste de fase) con arreglo al método recomendado por la Organización Mundial de la Salud en 1997 o por cualquier otro método que dé resultados equivalentes (17).
En función del país y de la técnica analítica empleada se establecen unos valores límites ambientales. La normativa española establece que ningún trabajador estará expuesto a una concentración de amianto en el aire superior al valor límite ambiental de exposición diaria (VLA-ED) de $0.1 \mathrm{f} / \mathrm{cm}^{3}$ medidas como una media ponderada en el tiempo para un período de ocho horas diarias tal como se especifica en el RD 396/2006 (18).

\section{DEMOLICIÓN, RETIRADA Y MANTENIMIENTO DE MATERIALES CON AMIANTO. RESIDUOS Y GESTIÓN DE LOS MISMOS}

Las actuaciones a realizar sobre los MCAs pueden variar desde la total eliminación hasta la no intervención, pues no siempre el diagnóstico recomienda la eliminación del amianto del edificio. La demolición de un edificio no puede iniciarse si no se han extraído primero todos los materiales que contengan amianto. Las operaciones de trabajo con amianto dependerán de si el MCA es friable o no friable.

Cuando se vayan a realizar actividades en las que los trabajadores estén expuestos a las fibras de amianto, o sean susceptibles de estarlo, se debe hacer un plan de trabajo. Los planes de trabajo se regulan en el RD 396/2006 por el que se establecen las disposiciones mínimas de seguridad y salud aplicables a los trabajos con riesgo de exposición al amianto. Su ámbito de aplicación comprende todas aquellas operaciones y actividades en las que los trabajadores estén expuestos o sean susceptibles de estar expuestos a fibras de amianto o de materiales que lo contengan y, especialmente, en trabajos de demolición de construcciones, desmantelamiento de maquinaria, retirada de amianto de instalaciones o estructuras, o trabajos de mantenimiento o reparación con riesgo de desprendimiento de fibras de amianto, así como el transporte y destrucción de residuos que contengan amianto.

En el Artículo 10.2 del RD 396/2006 se indica que antes del comienzo de las obras de demolición o mantenimiento los empresarios deberán adoptar todas las medidas adecuadas para identificar los materiales que puedan contener amianto. La identificación deberá quedar reflejada en el estudio de seguridad y salud a que se refiere el RD 1627/1997 (19), por el que se establecen las disposiciones mínimas en el estudio de seguridad y salud o en el estudio básico de seguridad y salud en las obras de construcción.

El artículo 11 del RD 396/2006 establece que el plan de trabajo debe prever la 
eliminación de los materiales con amianto antes de aplicar las técnicas de demolición salvo que ello cause un riesgo aún mayor que si se dejaran in situ, y las medidas necesarias para garantizar la seguridad y salud de los trabajadores que vayan a llevar a cabo estas operaciones, indicando si es friable el tipo de material a intervenir, su ubicación, y los procedimientos y medidas preventivas para limitar la dispersión de fibras en el ambiente y limitar la exposición de los trabajadores al amianto, entre otras especificaciones.

Las empresas que realizan trabajos de manipulación de amianto deben están inscritas en el RERA (Registro de Empresas con Riesgo de Amianto). Estas empresas deberán redactar y presentar el plan de trabajo (20). Conforme al artículo 12 del RD 396/2006, el plan de trabajo se debe presentar para su aprobación ante la autoridad laboral correspondiente al lugar de trabajo en el que vayan a realizarse tales actividades, recabándose en la tramitación de expediente el informe de la Inspección de Trabajo y Seguridad Social y de los órganos técnicos en materia preventiva de la correspondiente comunidad autónoma.

La tipología de los planes de trabajo depende de si suponen un trabajo específico para una operación o son de carácter general, y del tipo de material (friable o no friable) sobre el que se va a trabajar (21).

\subsection{Deconstrucción de MCAs}

\subsubsection{Trabajos de deconstrucción sobre material no friable}

El caso más frecuente de presencia de materiales con amianto en la edificación es el del fibrocemento (22). El amianto en este caso está mezclado con cemento, lo que convierte a éste en un material poco friable. La posible liberación de fibras de amianto al ambiente se puede producir por el envejecimiento de la placa por los agentes atmosféricos, o por la acción mecánica sobre las mismas. Si la manipulación del fibrocemento requiere necesariamente la rotura del mismo como único método de trabajo, se le dará la consideración de material friable y se trabajará con él como tal.

La mayoría de los materiales con fibrocemento se encuentra en cubiertas de naves industriales, depósitos y tuberías, y paredes fluviales. Normalmente se encuentran colocados superpuestos unos a otros mediante un pasante o fijador metálico. En las cubiertas de fibrocemento, por su baja friabilidad, la retirada de las placas es una de las operaciones menos complicadas sobre los MCAs. Hay que considerar, no obstante, que su manipulación implica la posibilidad de emisión de fibras, para lo que es necesaria la adopción de medidas de protección. Los trabajadores deben llevar mascarillas autofiltrantes FFP3 o mascarillas con filtros contra partículas tipo P3 y monos de trabajo desechables con capucha. Para la retirada de las cubiertas se deben desmontar los ganchos de anclaje, desatornillando la sujeción o cortándolos con las herramientas recomendadas según el tipo de operación, evitando máquinas rotativas por la elevada emisión de polvo que pueden generar (23). Tras quitar las placas, éstas se deben depositar con precaución, embalar con plástico de suficiente resistencia mecánica para evitar su rotura y señalizar con el símbolo del amianto. Si las placas están rotas, se han de humedecer con una impregnación encapsulante, retirarse con precaución y depositarse en un saco de residuo debidamente etiquetado. Una vez desmontadas las placas se debe proceder a la limpieza de toda la estructura de apoyo de la cubierta, por aspiración con filtros absolutos.

Existen otros MCAs no friables como las placas de falso techo o los suelos de PVC reforzados con amianto. Igual que con el fibrocemento, es necesario adoptar medidas de protección que eviten la inhalación de fibras debido a la manipulación de las placas y losetas vinílicas. Éstas se deben extraer con herramientas de corte o golpeado manual que desprendan las mínimas fibras al ambiente, evitando en lo posible cualquier herramienta de fricción o abrasión. Tras quitar las placas y losetas, éstas se deben depositar con precaución, embalar con plástico y señalizar con el símbolo del amianto.

\subsubsection{Trabajos de deconstrucción sobre material friable}

El desamiantado del ignifugante de la estructura metálica de un edificio es uno de los casos de desamiantado más complejo, debido a la friabilidad del material y a su disposición (22). Se trata de una mezcla de diferentes productos, entre los que pueden encontrarse tierras de diatomeas, material de fraguado, fibras de celulosa y fibras de amianto, entre otros. Debido a que un desamiantado mal realizado provoca una contaminación ambiental por fibras de amianto mucho más elevada que la debida a la presencia del material de amianto, es imprescindible la adopción de un protocolo de trabajo adecuado que garantice una mínima emisión de fibras a la zona de trabajo y evite su salida al exterior. Los trabajadores que realizan estos trabajos deben 
disponer de dispositivos filtrantes, contra partículas, de ventilación asistida con máscara, TMP3, o capuchas THP3 y mono de trabajo desechable con capucha.

Primero se deben realizar una serie de trabajos preliminares como la retirada del mobiliario y luminarias y el aislamiento y confinamiento de la zona de trabajo cerrando las entradas de aire para trabajar en depresión, entre 10 y $20 \mathrm{~Pa}$, utilizando un sistema de filtración de aire. La extracción debe funcionar de manera permanente con posterioridad a la finalización de los trabajos, para asegurar la limpieza de la zona de trabajo, la cual debe disponer de un túnel de acceso para el personal y otro para el material.

El método de trabajo escogido debe limitar la exposición de los trabajadores a las fibras de amianto durante las operaciones de retirada, rascado y limpieza. Existen diversas técnicas como la aspiración directa del amianto proyectado y después rascado por vía húmeda de las zonas restantes, humidificación y rascado manual acompañado de recogida de los residuos y aspiración y rascado mecánico con captador del material en la fuente, mediante aspiración. En todos los casos es deseable utilizar un agente impregnante que baje de forma significativa la generación de polvo y que, preferentemente, sea coloreado para permitir controlar que ha penetrado bien en el corazón de los materiales con amianto. Una vez eliminado el amianto, se inspeccionará para comprobar si quedan MCAs, se aspirará y limpiarán paredes, techos y suelo y se dejará en funcionamiento la depresión 48 horas más para asegurar la limpieza. Antes de la retirada de los plásticos que aíslan la zona de trabajo es aconsejable la aplicación de un fijador sobre la superficie de estos.

Otros materiales friables existentes en las edificaciones son los calorifugados y los materiales aislantes. Los calorifugados es habitual encontrarlos en la reparación de tuberías de agua caliente o en la retirada de material aislante con amianto. Los aislantes pueden encontrarse ante trabajos de mantenimiento en turbinas de centrales eléctricas o desmantelamiento de las instalaciones. Como en el caso de los ignifugados, es imprescindible la adopción de un protocolo de trabajo adecuado que garantice una mínima emisión de fibras a la zona de trabajo y evite su salida al exterior.

\subsection{Residuos y almacenamiento}

El amianto está considerado por la Unión Europea como residuo especial y como tal debe ser gestionado (24). En la normativa europea se cataloga a los residuos de amianto dentro de la partida "17 0605 Materiales de construcción que contienen amianto". Las directivas europeas sobre residuos peligrosos han sido incorporadas progresivamente a las normativas de los diferentes estados miembros.

La gestión de los residuos de amianto es una de las asignaturas pendientes de la industria de la construcción. Por la peligrosidad del amianto deben extremarse las medidas no sólo en el vertido sino también en el embalado y el transporte. EI RD 105/2008 (25), además de todos los requisitos aplicables a la gestión de residuos generados en obras de construcción o demolición de edificios y de ingeniería civil, establece que aquellas operaciones susceptibles de estar expuestas a fibras de amianto se regirán también por el RD 396/2006.

Los residuos de amianto o que contengan amianto, incluidos los resultantes de operaciones de limpieza y mantenimiento, deben recogerse en recipientes herméticos y adecuadamente etiquetados indicando que contienen amianto. Los contenedores con residuos de amianto han de transportarse lo más rápidamente posible fuera de la zona de trabajo y se depositarán en vertederos de residuos peligrosos.

Los residuos de materiales con amianto pueden disponerse finalmente como:

a. Relleno de seguridad: se disponen en doble bolsa de $200 \mu \mathrm{m}$, con lavado externo antes del transporte. El transporte se realiza en contenedores cerrados de apertura lateral. Este es un sistema poco económico.

b. Fusión: consiste en fundir el amianto para transformarlo en vidrio. El proceso es caro y la tecnología asociada compleja.

c. Encapsulado: se introduce el amianto en una estructura que no permita su migración al medio ambiente.

d. Gresificación: el amianto es empleado como materia prima, transformándose a lo largo del proceso.

Debido a la prohibición total del amianto, la industria ha ido incorporando materiales sustitutivos al mismo. La sustitución del amianto por otro material debe valorarse a dos niveles. En primer lugar, debe considerarse siempre la problemática que genera la sustitución directa del mismo cuando ha sido profusamente empleado y se decide eliminarlo. En segundo lugar, el material alternativo debe ser seguro en cuanto a su extracción, fabricación y uso. 


\section{IDENTIFICACIÓN DE MINERALES DE AMIANTO MEDIANTE TÉCNICAS INSTRUMENTALES}

Tal como se ha indicado en apartados anteriores, en España sólo están acreditados los laboratorios para el análisis de fibras y no existe una acreditación específica para analizar amianto en materiales. Para la identificación de las diferentes variedades de amianto, las técnicas más empleadas son la microscopía óptica, la microscopía electrónica de barrido y la difracción de rayos $X$.

\subsection{Identificación mediante microscopía óptica (MO)}

En el año 2010, dentro de los Métodos de toma de muestras y análisis, el Instituto de Seguridad e Higiene en el Trabajo editó un Documento en el que se describe el protocolo encaminado a la determinación cualitativa (Identificación) de fibras de amianto mediante MO (26). El método consiste básicamente en dos etapas:

En la primera etapa se examina el material de una muestra representativa con el microscopio estereoscópico para localizar, seleccionar y separar las fibras de la matriz que serán montadas sobre un portamuestras con líquido dispersante de un determinado índice de refracción. Esta etapa debe considerarse como una pre-identificación, aunque observando la morfología y ciertas propiedades físicas se obtiene una información bastante segura del tipo de amianto. Las fotos de las Figuras 4 y 5 corresponden a materiales de fibrocemento y muestran el aspecto del crisotilo y la crocidolita respectivamente.

En una segunda etapa se observan las preparaciones obtenidas en la primera etapa con el microscopio de polarización estudiando su morfología y sus propiedades ópticas (color, pleocroísmo, birrefringencia, colores de interferencia, extinción, signo de la elongación, etc.

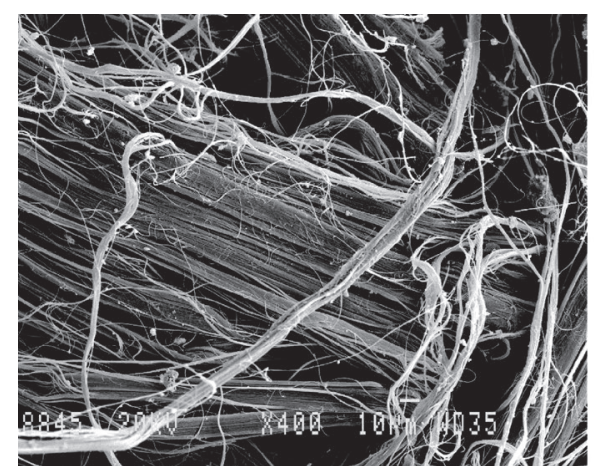

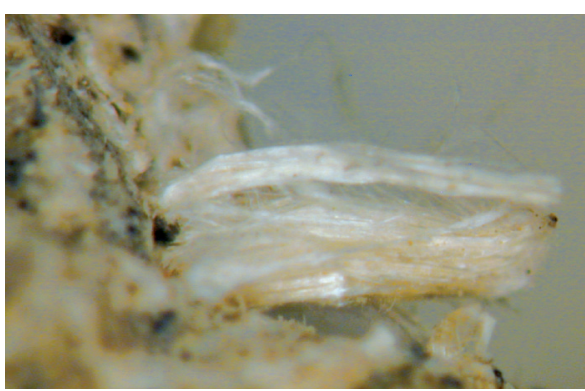
4

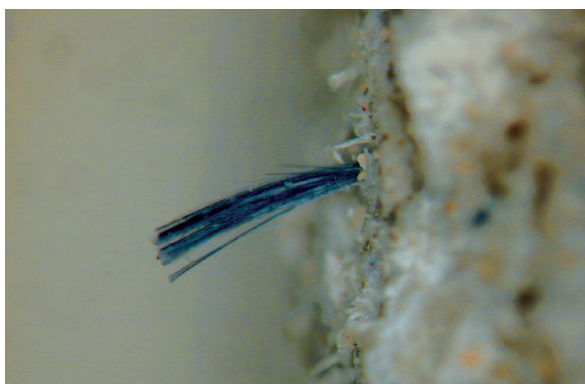

La identificación será positiva si las propiedades observadas en las fibras se corresponden con las de la estructura cristalina de alguno de los minerales de amianto.

\subsection{Identificación mediante microscopía electrónica de barrido (MEB)}

La MEB presenta, por comparación con la $\mathrm{MO}$, algunas ventajas y también algunas desventajas en la identificación de los minerales de amianto.

La desventaja más importante es que no se pueden aprovechar las propiedades ópticas de los minerales, entre ellas la más determinante que es el color.

Por el contrario, las muestras pueden observarse a grandes aumentos obteniendo fotografías de mucho más detalle. Además, los minerales se pueden analizar por dispersión de energías (EDAX) para comprobar la composición.

En las Figuras 6 y 7 vienen reflejadas la morfología y la composición de una muestra de crisotilo y otra de amosita.
4. Aspecto del crisotilo en el microscopio óptico a 50 aumentos.

5. Aspecto de la crocidolita en el microscopio óptico a 50 aumentos.

6. Imagen al microscopio electrónico y microanálisis de una muestra de crisotilo

7. Imagen al microscopio electrónico y microanálisis de una muestra de amosita 
8. Difractograma de una muestra de fibrocemento con crisotilo y crocidolita
El hecho de encontrar oro -Au- en los análisis indica que las muestras han sido metalizadas para posterior estudio mediante electrones secundarios. En general nosotros preferimos este tipo de análisis puesto que la calidad de las fotos es muy superior. En ocasiones, sin embargo, utilizamos electrones retrodispersados para lo que no es preciso metalizar las muestras y aunque la calidad de las fotos sea inferior, es más preciso el análisis ya que no se añade al espectro de EDAX el elemento propio del metalizado.

\subsection{Identificación mediante difracción de rayos $X$ (DRX)}

La DRX es una técnica de análisis estructural que se basa en que cada compuesto cristalino, por el hecho de serlo, presenta una respuesta específica a los rayos $\mathrm{X}$. De este modo, cotejando los diferentes picos (más precisamente espaciados reticulares) que componen un determinado difractograma con los que están tabulados podemos identificar con precisión el compuesto o compuestos que forman la muestra.

Por lo expresado en el apartado anterior, la DRX es la técnica más adecuada también para la identificación de minerales de amianto aunque hay que hacer algunas consideraciones:

1. En general, para obtener una respuesta de un determinado compuesto por DRX es necesaria una cierta concentración.

2. Además hay compuestos más cristalinos (con más intensidad en la respuesta por DRX) y menos cristalinos (de respuesta menos intensa). Los minerales de amianto por su aspecto fibroso son difíciles de triturar y su respuesta en DRX no es demasiado intensa.

3. Teniendo en cuenta que los minerales de amianto normalmente están englobados dentro de otros materiales (por ejemplo cemento en el caso más general), es necesario concentrarlos eliminando los materiales accesorios.

4. Para concentrar los minerales de amianto, por ejemplo en muestras de fibrocemento, recomendamos hacerlo por tamizado. La idea de eliminar con una disolución de $\mathrm{HCl}$ la portlandita y la calcita resultante del proceso de carbonatación no nos parece adecuada porque aunque los minerales de amianto son silicatos, quedan afectados con ese tipo de tratamiento.

En la Figura 8 se representa un difractograma correspondiente a una muestra de fibrocemento en la que después de concentrar por tamizado los minerales de amianto se identifican crisotilo y crocidolita.

\section{CONCLUSIÓN}

La preocupación de las administraciones por los riesgos para la salud pública asociados con la inhalación de las fibras de amianto se ha traducido en la prohibición de sus distintos usos en los países desarrollados, mientras en los países en vías de desarrollo los procesos de extracción, procesado y uso del amianto continúan.

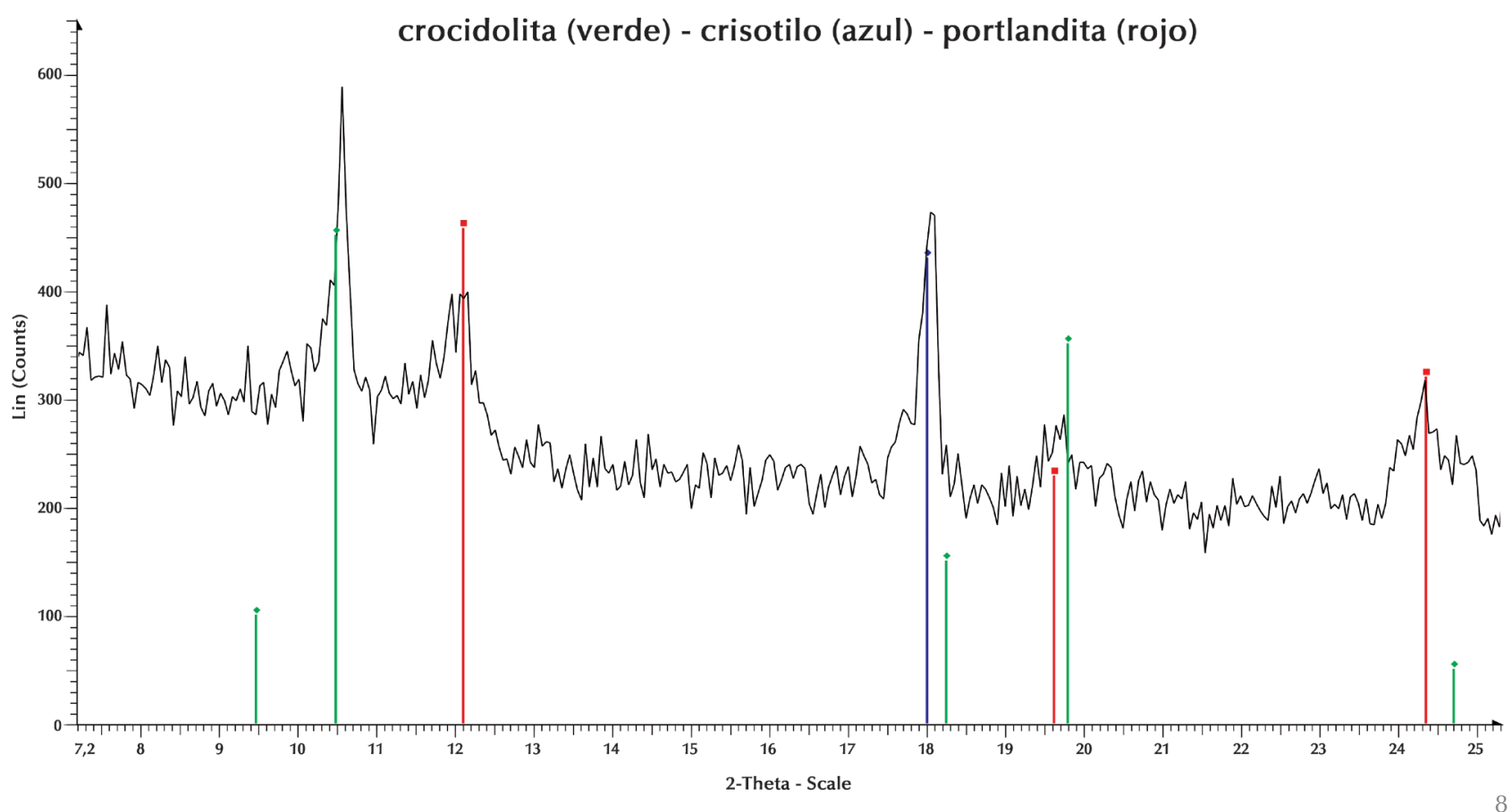


A pesar de su prohibición, el problema reside en el amianto ya instalado y el que se encuentra en forma de residuo. La presencia de MCAs en un edificio no supone directamente una situación de riesgo. Determinar la presencia o no de amianto en los edificios es fundamental para la salud de las personas que los ocupan, así como para los trabajadores que intervengan en su mantenimiento, reparación o derribo por la elevada contaminación ambiental que se puede producir al manipularlos.

El proceso de diagnosis de los edificios existentes debe permitir escoger el sistema de intervención más apropiado en cada caso.

Con los resultados del proceso se redacta un informe que debe establecer las recomendaciones más adecuadas en función del resultado de la diagnosis, concluyendo con la necesidad o no de realizar algún tipo de intervención.
Respecto de la identificación de MCAs existe consenso en la utilización de técnicas instrumentales de caracterización estructural. Por nuestra propia experiencia la difracción de rayos $X$ podría ser la técnica más adecuada, siempre que se hagan tratamientos de las muestras que concentren el amianto, evitando así los problemas de límite de detección que presenta esta técnica. Las técnicas de microscopía óptica y microscopía electrónica de barrido se utilizan también para determinar el tipo de mineral de amianto y, además, son insustituibles si se trata de contabilizar fibras en estudios de calidad del aire.

\section{AGRADECIMIENTOS}

El presente trabajo se enmarca dentro del Proyecto MES INNPACTO (IPT-3800002010-16) del Plan Nacional de I+D+l del Ministerio de Ciencia e Innovación, financiado con fondos FEDER.

\section{REFERENCIAS}

(1) Graus, R.; Trujillo, L.; Villaverde, M.; Freixa, A.; Ferrer; J.; Dirección de Perdigó, M. y Casanovas, X. (2001). Prospección sobre la presencia de amianto o de materiales que lo contengan en edificios. Informe. p. 197, Fundación para la Prevención de Riesgos Laborales. Institut d'Estudis de la Seguretat. Col·legi d'Aparelladors i Arquitectes Tècnics de Barcelona, Barcelona.

(2) Directiva 83/477/CEE, del Consejo, de 19 de septiembre de 1983, sobre la protección de los trabajadores contra los riesgos relacionados con la exposición al amianto durante el trabajo. Modificada por la Directiva 91/382/CEE de 25 de junio de 1991 y por la directiva 2003/18/CE de 27 de marzo de 2003

(3) Directiva 76/769/CEE del Consejo, de 27 de Julio de 1976, relativa a la aproximación de las disposiciones legales, reglamentarias y administrativas de los Estados Miembros que limitan la comercialización y el uso de determinadas sustancias y preparados peligrosos. Modificada por las Directiva 83/477/CEE, 85/610/CEE, 91/659/CEE, 94/60/CE, 97/56/CE y 1999/77/CE.

(4) Harris, L. V. (2003). Asbestos: old foe in 21 st century developing countries. The Science of the Total Environment, 307(1-3): 1-9. doi:10.1016/S0048-9697(02)00504-1.

(5) Casanovas, X.; Trujillo, L.; Freixa, A. (2003). Detección de amianto en edificios (II): Identificación y metodología de análisis. Nota Técnica de Prevención nº 633, Instituto Nacional de Seguridad e Higiene en el Trabajo INSHT, Barcelona.

(6) ICSC: 0014. International Chemical Safety Cards: Chrysotile.

(7) ICSC: 1314. International Chemical Safety Cards: Crocidolite.

(8) Hueto, J., Almudevar, E. (2005) Análisis y detección de fibras en tejido pulmonar. Anales del Sistema Sanitario de Navarra, 28(1): 13-19.

(9) Artieda L., Beloqui A., Lezaun M. (2005) Cohorte poblacional de trabajadores expuestos a amianto. Navarra 1999-2004. Anales del Sistema Sanitario de Navarra, 28(3): 335-344.

(10) Casanovas, X., Trujillo, L., Freixa, A. (2003) Detección de amianto en los edificios (I): aspectos básicos. Nota Técnica de Protección, n 632, Instituto Nacional de Seguridad e Higiene en el Trabajo INSHT, Barcelona.

(11) EPA 340/1-90-019: 12/90. Asbestos/NESHAP Regulated Asbestos Containing Materials Guidance. EPA (U.S. Environmental Protection Agency), 1990.

(12) EPA AHERA Regulations 40 CFR 763 subpart E: Asbestos-Containing Materials in Schools. EPA (U.S. Environmental Protection Agency).

(13) HSE Asbestos: The survey guide. HSG264 Health and Safety Executive Books, Sudbury, 2010.

(14) NF X46-020. Diagnostic amiante. Repérage des matériaux et produits contenant de I'amiante dans les immeubles bátis. AFNOR Association Francaise de Normalisation, 2002. 
(15) Freixa, A.; de Montserrat, J.; Colomer, J. (2005). Diagnóstico de amianto en edificios (II): Norma NF X46-020 (AFNOR). Nota Técnica de Prevención, n. ${ }^{\circ} 708$, Instituto Nacional de Seguridad e Higiene en el Trabajo INSHT, Barcelona.

(16) Graus, R. (1998). Manual per a la diagnosi i el tractament de l'amiant a la construcció. p. 42-45, Col·legi d'Aparelladors i Arquitectes Tècnics de Barcelona, Barcelona.

(17) Determinación de la concentración de fibras en el aire. Método recomendado: microscopía óptica con dispositivo para contraste de fase (método de filtros de membrana). OMS, Ginebra, 1997.

(18) REAL DECRETO 396/2006, de 31 de marzo, por el que se establecen las disposiciones mínimas de seguridad y salud aplicables a los trabajos con riesgo de exposición al amianto, 2006.

(19) REAL DECRETO 1627/1997, de 24 de octubre, por el que se establecen disposiciones mínimas de seguridad y salud en las obras de construcción, 1997.

(20) Calleja, A., Hernández, S., Freixa, A. (2008). Amianto: planes de trabajo para operaciones de retirada o mantenimiento. Nota Técnica de Prevención, n. ${ }^{\circ}$ 796, Instituto Nacional de Seguridad e Higiene en el Trabajo INSHT, Barcelona.

(21) Calleja, A., Hernández, S., Freixa, A. (2008). Amianto: planes de trabajo con amianto: orientaciones prácticas para su realización. Nota Técnica de Prevención, n. ${ }^{\circ}$ 815, Instituto Nacional de Seguridad e Higiene en el Trabajo INSHT, Barcelona.

(22) Calleja, A., Hernández, S., Freixa, A. (2010). Operaciones de demolición, retirada o mantenimiento de materiales con amianto. Ejemplos prácticos. Nota Técnica de Prevención, n. ${ }^{\circ}$ 862, Instituto Nacional de Seguridad e Higiene en el Trabajo INSHT, Barcelona.

(23) UNE 88-411-87. Productos de Amianto Cemento. Directrices para su corte y mecanizado en obra. AENOR, Madrid, 1999.

(24) Directiva 91/689/CE y Decisión 2001/573/CE de la Unión Europea relativa a los residuos peligrosos. Incorporada al ordenamiento jurídico español mediante el Real Decreto 952/1997, de 20 junio que modifica el Reglamento para la ejecución de Ley 20/1986, de 14 de mayo, Básica de Residuos Tóxicos y Peligrosos, aprobado mediante Real Decreto 833/1988, de 20 de julio.

(25) REAL DECRETO 105/2008, de 1 de febrero, por el que se regula la producción y gestión de los residuos de construcción y demolición, 2008.

(26) MTA/PI - 010/A09 Determinación cualitativa (Identificación) de fibras de amianto en materiales. Método de polarización-dispersión/Microscopía óptica. Instituto Nacional de Seguridad e Higiene en el Trabajo, 2010. 